\title{
A Theorem of Tits, Normalizers of Maximal Tori and Fibrewise Bousfield-Kan Completions
}

\author{
By
}

\author{
Frank NeUmanN*
}

\begin{abstract}
We use a theorem of Tits on the presentation of the normalizer of a maximal torus of a connected compact semisimple Lie group in terms of generators and relations to give several equivalent conditions for the splitting of the associated normalizer group extension and interprete them in terms of $p$-adic fibrewise homotopy theory.
\end{abstract}

\section{§0. Introduction}

The isomorphism type of a compact connected semisimple Lie group $G$ is completely determined by the isomorphism type of the normalizer of the maximal torus $T(G)$ as it was shown by Curtis, Wiederhold and Williams [C-W-W]. This was generalized much later by Notbohm for any compact connected Lie group [N]. In their classical paper Curtis, Wiederhold and Williams also studied the related question when the group extension

$$
0 \rightarrow T(G) \rightarrow N(G) \stackrel{\pi}{\rightarrow} W(G) \rightarrow 1
$$

is a split extension and the normalizer $N(G)$ is completely determined by the action of the Weyl group $W(G)$ on the maximal torus $T(G)$. Using a theorem of Tits [T2] giving an explicit description of the normalizer in terms of generators and relations, they could decide case-by-case for which simple Lie groups the above normalizer sequence is split exact.

In this note we use the theorem of Tits to interprete the splitting of the above normalizer group extension in terms of fibrewise $p$-adic Bousfield-Kan completion $[\mathrm{B}-\mathrm{K}]$ of the associated fibration of classifying spaces. It turns

Communicated by K. Saito, November 9, 1999.

1991 Mathematics Subject Classification. 20 F 55, 20 G 20, 20 J 06, 22 E 15, 57 T 10

* Mathematisches Institut, University of Göttingen, Bunsenstr. 3-5, D-37073 Göttingen, Germany

e-mail: neumann@cfgauss.uni-math.gwdg.de 
out, that the splitting question only has to be considered locally at the prime 2. Namely, we have

Theorem. Let $G$ be a compact connected Lie group. The group extension

$$
0 \rightarrow T(G) \rightarrow N(G) \stackrel{\pi}{\rightarrow} W(G) \rightarrow 1
$$

is a split extension if and only if the fibration

$$
(\mathbb{Z} / 2)_{\infty} B T(G) \rightarrow(\mathbb{Z} / 2)_{\infty}^{\circ} B N(G) \stackrel{B \pi}{\rightarrow} B W(G)
$$

has a section, i.e. is fibre homotopy equivalent to the fibration

$$
(Z / 2)_{\infty} B T(G) \rightarrow E W(G) \times_{W(G)}(Z / 2)_{\infty} B T(G) \rightarrow B W(G) .
$$

Moreover, it turns out that for odd primes $p$ the fibrewise $p$-adic completed fibration of classifying spaces

$$
(\mathbb{Z} / p)_{\infty} B T(G) \rightarrow(\mathbb{Z} / p)_{\infty}^{\circ} B N(G) \stackrel{B \pi}{\rightarrow} B W(G)
$$

has always a section.

Here we will consider only the Lie group case, but it is interesting to ask if it is possible to derive a similar homotopy theoretic interpretation in the case of a split semisimple reductive algebraic group $G$ over a field $k$, where the theorem of Tits is also known [T2].

\section{§1. A Theorem of Tits and the Splitting of the Normalizer of a Maximal Torus of a Compact Connected Lie Group}

Let $G$ be a compact connected semisimple Lie group. Fix a maximal torus $T(G)$ of $G$ and let $N(G)$ be the normalizer of $T(G)$ in $G$. The Weyl group of $G$ is given by $W(G)=N(G) / T(G)$. First we recall some basic facts from Lie group theory [B-tD]. The normalizer $N(G)$ acts on $T(G)$ by conjugation

$$
N(G) \times T(G) \rightarrow T(G), n \cdot t=n t n^{-1} .
$$

The action restricted to $T(G)$ is trivial, so factors through the quotient $W(G)$ $=N(G) / T(G)$ inducing an action of $W(G)$ on $T(G)$

$$
W(G) \times T(G) \rightarrow T(G), n \cdot T(G)=n t n^{-1} .
$$


We will study the group extension

$$
0 \rightarrow T(G) \rightarrow N(G) \stackrel{\pi}{\rightarrow} W(G) \rightarrow 1
$$

and derive several equivalent conditions under which this extension is a split extension so that

$$
N(G) \cong T(G) \rtimes W(G) .
$$

Denote by $L(G)$ the Lie algebra of $G$ which we identify with the tangent space to $G$ at the identity element $e$ of $G$. Formally we may split $L(G) \cong L(T(G)$ ) $\oplus L(G / T(G))$, where $L(G / T(G))$ is the orthogonal complement of $L(T(G)$ ) with respect to the $W(G)$-invariant inner product on $L(T(G))$.

Let $\Psi(G)$ be the set of roots and $\Psi^{0}(G)$ be a simple root system of $G$. For any root $\alpha \in \Psi^{0}(G)$ let $s_{\alpha}$ be the corresponding reflection of $L(T(G))$. The Weyl group $W(G)$ has a presentation as a finite real reflection group with generators the set of reflections $S=\left\{s_{\alpha}: \alpha \in \Psi^{0}(G)\right\}$ subject to the relations

(1) $s_{\alpha}^{2}=1$

(2) $\left(s_{\alpha} s_{\beta}\right)^{m_{\alpha \beta}}=1$ for all $\alpha \neq \beta$ and $m_{\alpha \beta} \in\{2,3,4,6\}$

The $W(G)$-invariant inner product allows us to identify the $W(G)$ representation $L(T(G))$ with the dual representation $L(T(G))^{*}=\operatorname{Hom}_{\mathbb{R}}(L(T(G))$, $\boldsymbol{R})$. Denote this isomorphism by $\kappa: L(T(G)) \rightarrow L(T(G))^{*}$.

For $\alpha \in \Psi(G)$ and $x \in L(T(G))$ we have

$$
s_{\alpha}(x)=x-\langle\alpha, x\rangle \alpha^{*}
$$

where $\alpha^{*}=2 \alpha /\langle\alpha, \alpha\rangle$ is the coroot corresponding to $\alpha$. For the set of coroots of $G$ let us write $\Psi(G)^{*}=\left\{\alpha^{*}: \alpha \in \psi(G)\right\}$. If it becomes necessary to distinguish between $L(T(G))$ and $L(T(G))^{*}$ we will denote by $\alpha^{\wedge}=\kappa\left(\alpha^{*}\right) \in L(T(G))^{*}$, the inverse root of $\alpha$. Let $\exp : L T(G) \rightarrow T(G)$ be the exponential map and set $I=\operatorname{ker}(\exp )$ and $I^{*}=\left\{\alpha \in L\left(T(G)^{*}: \alpha I \subset Z\right\} . \quad I\right.$ is called the integral lattice and $I^{*}$ the lattice of integral forms. We get the following commutative diagram 


$$
\begin{aligned}
& 0 \rightarrow \mathbb{Z}^{n} \rightarrow \quad \mathbb{R}^{n} \quad \stackrel{\exp }{\rightarrow} T(G) \rightarrow 1
\end{aligned}
$$

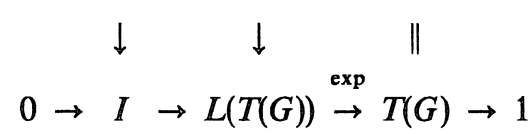

$$
\begin{aligned}
& \begin{array}{lll}
\downarrow & \downarrow^{\alpha} & \downarrow^{\theta_{\alpha}} \\
0 \rightarrow \boldsymbol{Z} \rightarrow \quad & \boldsymbol{R} & \stackrel{e}{\rightarrow} U(1) \rightarrow 1
\end{array}
\end{aligned}
$$

where $e: R \rightarrow U(1)$ is given by $t \mapsto \exp (2 \pi i t)$ and $\theta_{\alpha}: T(G) \rightarrow U(1)$ is defined by $\exp (H) \mapsto \exp (2 \pi i \alpha(H))$ as the global root corresponding to $\alpha \in L(T(G))^{*}$. It is easy to see that $\Psi(G) \subset I^{*}$ and $\Psi(G)^{*} \subset I$ [B-tD].

For every root $\alpha \in \Psi(G)$ we now define an element $h_{\alpha} \in T(G)$ by $h_{\alpha}=\exp \left(\frac{1}{2} \alpha^{\wedge}\right)$. We get immediately from the above considerations that $h_{\alpha}^{2}=1$ and $h_{\alpha}=h_{-\alpha}$.

The normalizer $N(G)$ of the maximal torus $T(G)$ also has a presentation with generators and relations like a braid group mixed with the toral part as given in the following theorem of Tits [T1]. See also [C-W-W]. In [T2] Tits proved a similar theorem in the case of a split semisimple reductive algebraic group $G$.

Theorem 1.1 (Tits). Let $G$ be a compact connected semisimple Lie group, $T(G)$ a maximal torus and $N(G)$ its normalizer in $G$. For every simple root $\alpha \in \Psi^{0}(G)$ there exists an element $q_{\alpha} \in N(G)$ such that

$$
\pi^{-1}\left(s_{\alpha}\right)=T(G) \cup q_{\alpha} T(G)
$$

with the following properties:

(1) $q_{\alpha}^{2}=h_{\alpha}$

(2) (Braid condition)

$q_{\alpha} q_{\beta} q_{\alpha} \cdots=q_{\beta} q_{\alpha} q_{\beta} \cdots$ for all $\alpha \neq \beta$ where the factors are repeated $m_{\alpha \beta}$ times, where $m_{\alpha \beta} \in\{2,3,4,6\}$.

(3) $q_{\alpha} t q_{\alpha}^{-1}=s_{\alpha}(t)$ for $t \in T(G)$.

Moreover, the normalizer $N(G)$ is isomorphic to the group generated by the set $T(G) \cup\left\{q_{\alpha}: \alpha \in \Psi^{0}(G)\right\}$ subject to the relations (1), (2), (3) and those coming from $T(G)$.

Let $T_{\infty}(G)$ be the subgroup of $T(G)$ of elements having finite order, called the discrete approximation of $T(G)$. We have $T_{\infty}(G) \cong(Q / Z)^{n}$ where $n$ is the rank of 
$T(G) . \quad T_{\infty}(G)$ is mapped to itself under the action of the Weyl group $W(G)$. For all $\alpha \in \Psi^{0}(G)$ the element $h_{\alpha}$ lies in $T_{\infty}(G)$. Let $N_{\infty}(G)$ be the subgroup of $N(G)$ generated by the set $T_{\infty}(G) \cup\left\{q_{\alpha}: \alpha \in \Psi^{0}(G)\right\}$ subject to the relations (1),(2), (3) in the theorem of Tits and those from $T_{\infty}(G)$. Then $N_{\infty}(G)$ is the discrete approximation of $N(G)$.

The inclusion $i: T_{\infty}(G) \rightarrow T(G)$ induces a homomorphism of group extensions

$$
\begin{aligned}
& 0 \rightarrow T_{\infty}(G) \rightarrow N_{\infty}(G) \stackrel{\pi}{\rightarrow} W(G) \rightarrow 1 \\
& \begin{array}{rlll}
\downarrow^{i} & & \downarrow & \| \\
0 & \rightarrow T(G) & \rightarrow & N(G) \stackrel{\pi}{\rightarrow} W(G) \rightarrow 1
\end{array}
\end{aligned}
$$

For a fixed prime $p$ let $T_{p^{\infty}}(G)$ denote the subgroup of $T_{\infty}(G)$ of elements having order a power of $p$. We have $T_{p^{\infty}}(G) \cong\left(Z / p^{\infty}\right)^{n}$ where $n$ is again the rank of $T(G)$ and $Z / p^{\infty}$ denotes the group $Z\left[\frac{1}{p}\right] / Z=\operatorname{colim}_{s} Z / p^{s}$. The Weyl group $W(G)$ acts on $T_{p^{\infty}}(G)$ and the inclusion

$$
j: \prod_{p \text { prime }} T_{p^{\infty}}(G) \stackrel{\cong}{\rightarrow} T_{\infty}(G)
$$

is a $W(G)$-equivariant isomorphism.

From the universal property of the product we get therefore a unique homomorphism $\pi_{p^{\infty}}: T_{\infty}(G) \rightarrow T_{p^{\infty}}(G)$ making the diagram

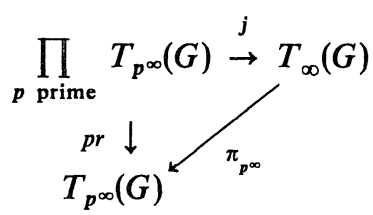

commutative. The map $\pi_{p^{\infty}}$ is also a $W(G)$-equivariant map.

Now let $N_{p^{\infty}}(G)$ be the subgroup of $N_{\infty}(G)$ generated by the set $T_{p^{\infty}}(G) \cup\left\{q_{\alpha}: \alpha \in \Psi^{0}(G)\right\}$ subject to the relations (1), (2), (3) in the theorem of Tits and those coming from $T_{p^{\infty}}(G)$. The homomorphism $\pi_{p^{\infty}}$ induces a homomorphism of group extensions 


$$
\begin{aligned}
& 0 \rightarrow T_{\infty}(G) \rightarrow N_{\infty}(G) \stackrel{\pi}{\rightarrow} W(G) \rightarrow 1 \\
& \downarrow \pi_{p^{\infty}} \quad \downarrow \quad \| \\
& 0 \rightarrow \mathbb{T}_{p^{\infty}}(G) \rightarrow N_{p^{\infty}}(G) \stackrel{\pi}{\rightarrow} W(G) \rightarrow 1
\end{aligned}
$$

Since $h_{\alpha}^{2}=1$ for all $\alpha \in \Psi^{0}(G)$ it follows that $h_{\alpha} \in T_{2 \infty}(G)$. Therefore for all odd primes $p$ we have $\pi_{p^{\infty}}\left(h_{\alpha}\right)=1$.

From this it follows immediately that for odd primes $p$ the group $N_{p^{\infty}}(G)$ is generated by the set $T_{p^{\infty}}(G) \cup\left\{q_{\alpha}: \alpha \in \Psi^{0}(G)\right\}$ subject only to the relations

(1) $q_{\alpha}^{2}=1$

(2) $\left(q_{\alpha} q_{\beta}\right)^{m_{\alpha \beta}}=1$ for all $\alpha \neq \beta$

(3) $q_{\alpha} t q_{\alpha}^{-1}=s_{\alpha}(t)$ for $t \in T(G)$.

Let $B_{p^{\infty}}(G)$ be the subgroup of $N_{p^{\infty}}(G)$ generated only by the set $\left\{q_{\alpha}: \alpha \in \Psi^{0}(G)\right\}$ subject to the relations (1'), (2'), (3'). The homomorphism $\pi: N_{p^{\infty}}(G) \rightarrow W(G)$ maps $B_{p^{\infty}}(G)$ isomorphically onto $W(G)$ since the elements $\pi\left(q_{\alpha}\right)=s_{\alpha}$ for $\alpha \in \Psi^{0}(G)$ generate the Weyl group $W(G)$ and $B_{p^{\infty}}(G) \cap T_{p^{\infty}}(G)=\emptyset$. Therefore we have shown the following theorem:

Theorem 1.2. Let $G$ be a compact connected semisimple Lie group. For each prime $p \neq 2$ the group extension

$$
0 \rightarrow T_{p^{\infty}}(G) \rightarrow N_{p^{\infty}}(G) \stackrel{\pi}{\rightarrow} W(G) \rightarrow 1
$$

is a split extension with $N_{p^{\infty}}(G) \cong T_{p^{\infty}}(G) \rtimes W(G)$.

In the special case that $h_{\alpha}=1$ for all $\alpha \in \Psi^{0}(G)$ we can derive a stronger result. Then the group $N(G)$ is generated by the set $T(G) \cup\left\{q_{\alpha}: \alpha \in \Psi^{0}(G)\right\}$ subject to the relations

(1) $q_{\alpha}^{2}=1$

(2) $\left(q_{\alpha} q_{\beta}\right)^{m_{\alpha \beta}}=1$ for all $\alpha \neq \beta$

(3) $q_{\alpha} t q_{\alpha}^{-1}=s_{\alpha}(t)$ for $t \in T(G)$.

and in the same way as above we get immediately $N(G) \cong T(G) \rtimes W(G)$. From this we can conclude the following equivalent algebraic conditions for the splitting of the normalizer exact sequence:

Theorem 1.3. Let $G$ be a compact connected semisimple Lie group. The 
following statements are equivalent:

(1) For every $\alpha \in \Psi^{0}(G)$ it is $h_{\alpha}=1$.

(2) The group extension

$$
0 \rightarrow T_{2 \infty}(G) \rightarrow N_{2 \infty}(G) \stackrel{\pi}{\rightarrow} W(G) \rightarrow 1
$$

splits, i.e. $N_{2^{\infty}}(G) \cong T_{2^{\infty}}(G) \rtimes W(G)$.

(3) The group extension

$$
0 \rightarrow T(G) \rightarrow N(G) \stackrel{\pi}{\rightarrow} W(G) \rightarrow 1
$$

splits, i.e. $N(G) \cong T(G) \rtimes W(G)$.

In [C-W-W] Curtis, Wiederhold and Williams used special elements in the Lie algebra $L(T(G))$ to check case-by-case for which simple Lie groups the normalizer sequence splits. This was also investigated earlier by Tits. He considered also groups obtained by quotienting out proper subgroups of centers. It turns out that for $S U(2 n+1), S U(2 n) / Z, S O(n), G_{2}$ the normalizers always split, while the normalizers of $S U(2 n), S p(n) / Z, \operatorname{Spin}(n), F_{4}, E_{6}, E_{7}, E_{8}$ and their quotients modulo centers $Z$ do not split.

\section{§2. Fibrewise Bousfield-Kan Completions and the Splitting of the Normalizer Sequence}

Now we will use fibrewise homotopy theory to interprete the algebraic conditions for the splitting of the normalizer of a maximal torus of a compact connected Lie group in topological terms. Let $R$ be a commutative ring. For a fibration of topological spaces

$$
F \rightarrow X \rightarrow B
$$

fibrewise $R$-completion in the sense of Bousfield-Kan [B-K] gives in functorial manner a fibration

$$
R_{\infty} F \rightarrow R_{\infty}^{\circ} X \rightarrow B
$$

where $R_{\infty} F$ is the $R$-completion of the fibre $F$. Let $p$ be a prime. We will use fibrewise Bousfield-Kan completion for the ring $R=Z / p$ and for simplicity will always write $F_{p}^{\wedge}=(Z / p)_{\infty} F$ and $X_{p}^{\circ}=(Z / p)_{\infty}^{\circ} X$. 
Consider the following diagram of group extensions and homomorphisms of group extensions for a compact connected Lie group $G$.

$$
\begin{aligned}
& 0 \rightarrow T(G) \rightarrow N(G) \stackrel{\pi}{\rightarrow} W(G) \rightarrow 1 \\
& \begin{array}{ccc}
\uparrow^{i} & \uparrow & \| \\
0 \rightarrow T_{\infty}(G) & \rightarrow & N_{\infty}(G) \stackrel{\pi}{\rightarrow} W(G) \rightarrow 1
\end{array} \\
& \downarrow \pi_{p^{\infty}} \quad \downarrow \quad \| \\
& 0 \rightarrow T_{p^{\infty}}(G) \rightarrow N_{p^{\infty}}(G) \stackrel{\pi}{\rightarrow} W(G) \rightarrow 1
\end{aligned}
$$

Applying the classifying space functor $B(?)$ and fibrewise $p$-adic completions of the rows for a fixed prime $p$ in the sense of Bousfield-Kan yields the following diagram of fibrations and maps of fibrations:

$$
\begin{array}{cccc}
B T(G)_{p}^{\wedge} & \rightarrow & B N(G)_{p}^{\circ} \stackrel{B \pi}{\rightarrow} B W(G) \\
\uparrow \simeq & & \uparrow \simeq & \| \\
B T_{\infty}(G)_{p}^{\wedge} & \rightarrow & B N_{\infty}(G)_{p}^{\circ} & \stackrel{B \pi}{\rightarrow} B W(G) \\
\downarrow & & & \\
& & \downarrow & \\
B T_{p^{\infty}}(G)_{p}^{\hat{p}} & \rightarrow & B N_{p^{\infty}}(G)_{p}^{\circ} & \stackrel{B \pi}{\rightarrow} B W(G)
\end{array}
$$

with fibrewise homotopy equivalences. We obtain therefore the following interpretation of theorem 1.2 in terms of fibrewise homotopy theory:

Theorem 2.1. Let $G$ be a compact connected Lie group. For each prime $p \neq 2$ the fibration

$$
B T(G)_{p}^{\wedge} \rightarrow B N(G)_{p}^{\circ} \stackrel{B \pi}{\rightarrow} B W(G)
$$

has a section, i.e is fibre homotopy equivalent to the fibration

$$
B T(G)_{p}^{\wedge} \rightarrow E W(G) \times_{W(G)} B T(G)_{p}^{\wedge} \rightarrow B W(G) .
$$

In other words

$$
B N(G)_{p}^{\circ} \simeq B(T(G) \rtimes W(G))_{p}^{\circ} \simeq E W(G) \times_{W(G)} B T(G)_{p}^{\wedge}
$$


where $E W(G)$ is a free acyclic $W(G)$-space.

Proof. First of all let $Z(G)$ be the center of $G$. The compact Lie group $G / Z(G)$ has maximal torus $T(G) / Z(G)$ and Weyl group $W(G)$ and the normalizer of the maximal torus is given by $N(G) / Z(G)$. We have a commutative diagram

$$
\begin{aligned}
& 0 \rightarrow \quad T(G) \quad \rightarrow \quad N(G) \quad \stackrel{\pi}{\rightarrow} W(G) \rightarrow 1 \\
& \downarrow^{i} \quad \downarrow \quad \| \\
& 0 \rightarrow T(G) / Z(G) \rightarrow N(G) / Z(G) \rightarrow W(G) \rightarrow 1
\end{aligned}
$$

So the top extension splits if the bottom one is a split extension. Therefore (see [B-tD], Theorem 7.1) we can always assume that $G$ is simply connected. But if $G$ is simply connected it is certainly semisimple (see [B-tD], Remark 7.13). So the theorem follows from the above considerations and theorem 1.2.

As an immediate consequence we get the following result in terms of group cohomology, which was also announced in [C-W-W], Appendix 2:

Corollary 2.2. Let $G$ be a compact connected Lie group $G$. Then

$$
H^{*}\left(B N(G), Z\left[\frac{1}{2}\right]\right) \cong H^{*}\left(B(T(G) \rtimes W(G)), Z\left[\frac{1}{2}\right]\right)
$$

or equivalently

$$
H^{*}\left(B N(G), Z_{2}^{\wedge}\right) \cong H^{*}\left(B(T(G) \rtimes W(G)), Z_{2}^{\wedge}\right) .
$$

For a compact Lie group $G$ and a fixed prime $p$ let $S_{p} W(G)$ denote a $p$-Sylow subgroup of $W(G)$ and $S_{p} N(G)$ the inverse image of $S_{p} W(G)$ in $N(G) . \quad S_{p} N(G)$ is called a p-normalizer of $N(G)$. Now we can state the main theorem of this section:

Theorem 2.3. Let $G$ be a compact connected Lie group and $p$ be a fixed prime. The following statements are equivalent:

(1) The group extension

$$
0 \rightarrow T_{p^{\infty}}(G) \rightarrow N_{p^{\infty}}(G) \rightarrow W(G) \rightarrow 1
$$


splits.

(2) The group extension

$$
0 \rightarrow T(G) \rightarrow S_{p} N(G) \rightarrow S_{p} W(G) \rightarrow 1
$$

splits.

(3) The fibration

$$
B T(G) \rightarrow B S_{p} N(G) \rightarrow B S_{p} W(G)
$$

has a section.

(4) The fibration

$$
B T(G)_{p}^{\wedge} \rightarrow B N(G)_{p}^{\circ} \rightarrow B W(G)
$$

has a section.

(5) The fibration

$$
B T_{p^{\infty}}(G) \rightarrow B N_{p^{\infty}}(G) \rightarrow B W(G)
$$

has a section.

Proof. That assertion (2) follows from (1) is an immediate consequence of the following commutative diagram combining a pullback and a pushout diagram:

$$
\begin{aligned}
& 0 \rightarrow T_{p^{\infty}}(G) \rightarrow N_{p^{\infty}}(G) \rightarrow W(G) \rightarrow 1 \\
& \begin{array}{l}
\| \\
0 \rightarrow T_{p^{\infty}}(G) \rightarrow S_{p} N_{p^{\infty}}(G)
\end{array} \stackrel{\uparrow}{\uparrow} S_{p} W(G) \rightarrow 1 \\
& 0 \rightarrow T(G) \rightarrow S_{p} N(G) \rightarrow S_{p} W(G) \rightarrow 1
\end{aligned}
$$

The assertion (3) follows at once from (2) by applying the classifying space functor $B(?)$ which sends a splitting homomorphism to a section.

To show that (4) follows from (3) suppose now that the fibration

$$
B T(G) \rightarrow B S_{p} N(G) \rightarrow B S_{p} W(G)
$$

has a section $\sigma$. After fibrewise $p$-adic Bousfield-Kan completion we still have a section in the completed fibration

$$
B T(G)_{p}^{\wedge} \rightarrow B S_{p} N(G)_{p}^{\circ} \rightarrow B S_{p} W(G) .
$$


Since the homomorphism in cohomology

$$
i^{*}: H^{3}\left(B W(G), \pi_{2}\left(B T(G)_{p}^{\wedge}\right)\right) \rightarrow H^{3}\left(B S_{p} W(G), \pi_{2}\left(B T(G)_{p}^{\wedge}\right)\right)
$$

is a monomorphism (see also $[\mathrm{N}]$ ) it follows that the fibration

$$
B T(G)_{p}^{\wedge} \rightarrow B N(G)_{p}^{\circ} \rightarrow B W(G)
$$

has a section as required.

Now we show that (5) follows from (4). Let $F$ be the homotopy fibre of the completion map

$$
\phi: B T_{p^{\infty}}(G) \rightarrow B T_{p^{\infty}}(G)_{p}^{\wedge} \simeq B T(G)_{p}^{\wedge}
$$

Then we have

$$
F=\operatorname{hofib}(\phi) \simeq K\left(\left(Q_{p}^{\wedge}\right)^{n}, 1\right)
$$

where $n$ is the rank of $T(G)$ and we get the following diagram of fibrations

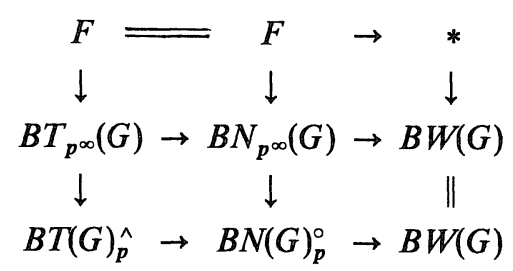

where the bottom fibration has a section. Obstruction theory (see [W]) shows that this section can be lifted to a section of the middle fibration, because

$$
H^{*+1}\left(B W(G), \pi_{*}(F)\right)=0
$$

since $W(G)$ is a finite group and $\operatorname{char}\left(Q_{p}^{\wedge}\right)=0$ (see [B]).

Finally we have to prove that (1) follows from (5). So suppose the fibration

$$
B T_{p^{\infty}}(G) \rightarrow B N_{p^{\infty}}(G) \rightarrow B W(G)
$$

has a section $\sigma$. The groups $T_{p^{\infty}}(G), N_{p^{\infty}}(G)$ and $W(G)$ are discrete groups and therefore the long exact sequence of homotopy groups degenerate to the following short exact sequence of groups

$$
0 \rightarrow \pi_{1}\left(B T_{p^{\infty}}(G)\right) \rightarrow \pi_{1}\left(B N_{p^{\infty}}(G)\right) \rightarrow \pi_{1}(B W(G)) \rightarrow 1
$$


which is nothing else than the group extension

$$
0 \rightarrow T_{p^{\infty}}(G) \rightarrow N_{p^{\infty}}(G) \rightarrow W(G) \rightarrow 1
$$

The section $\sigma$ induces a splitting homomorphism in the short exact sequence of fundamental groups and hence in the group extension.

We note that the same proof would apply also to an arbitrary extension of a torus $T$ by a finite group $H$

$$
0 \rightarrow T \rightarrow \stackrel{\pi}{\rightarrow} H \rightarrow 1
$$

and so the previous theorem remains true even in this more general situation.

As an immediate corollary we get finally from the algebraic considerations of the first section the main theorem as mentioned in the introduction:

Theorem 2.4. Let $G$ be a compact connected Lie group. The group extension

$$
0 \rightarrow T(G) \rightarrow N(G) \stackrel{\pi}{\rightarrow} W(G) \rightarrow 1
$$

is a split extension with $N(G) \cong T(G) \rtimes W(G)$ if and only if one and hence all of the statements in the previous theorem hold for the prime 2.

Explicit cohomological calculations of Andersen [A] show that a statement analogous to Theorem 2.1. is also true in the case of a connected p-compact group in the sense of Dwyer and Wilkerson [D-W], which are adequate homotopy theoretic replacements of compact Lie groups, having all the notions of maximal torus, Weyl group and normalizer. Andersen, instead of using the result of Tits, calculated the low-dimensional cohomology groups of the Weyl groups in all necessary cases. But it is still an open problem if in this framework there is an analogous statement of the theorem of Tits, giving an explicit description of the normalizer with generators and relations, which then would also allow to decide the splitting of the normalizer for any connected finite loop space with maximal torus [M-N] in the same manner as described in Theorem 2.4. In the case of $p$-compact groups the Weyl groups are p-adic pseudoreflection groups, so not real reflection groups anymore as in the Lie group case. The work of Broué, Malle and Rouquier [B-M-R] on the classification of complex reflection groups and their associated braid groups 
is closely related with this question.

\section{Acknowledgements}

We would like to thank J. M. Møller, D. Notbohm, L. Smith and A. Viruel for many useful discussions. Special thanks also to K. S. Andersen for telling me about his work.

\section{References}

[A] Andersen, K. S., Cohomology of Weyl Groups with Applications to Topology, Master thesis, University of Copenhagen (1997).

[B-K] Bousfield, A. K. and Kan, D. M., Homotopy Limits, Completions and Localizations, Springer Verlag New York, Lecture Notes in Math., 304 (1972).

[B-tD] Bröcker, T. and Dieck, T. tom, Representations of Compact Lie Groups, Springer Verlag, New York, Grad. Texts in Math., 98 (1985).

[B-M-R] Broué, M., Malle, G. and Rouquier, R., On complex reflection groups and their associated braid groups, in: B. N. Allison (ed.) et al: Representations of groups, CMS annual seminar Banff, Alberta, CMS Conf. Proc., 16 (1995), 1-13.

[B] Brown, K. S., Cohomology of Groups, Springer Verlag New York, Grad. Texts in Math., 87 (1982).

[C-W-W] Curtis, M., Wiederhold, A. and Williams, B., Normalizers of Maximal Tori, in: P. Hilton (ed.): Localizations in Group Theory and Homotopy Theory, Proc. of the Seattle Symposium 1974, Springer Verlag New York, Lecture Notes in Math., 418 (1974).

[D-W] Dwyer, W. G. and Wilkerson, C. W., Homotopy fixed point methods for Lie groups and finite loop spaces, Ann. of Math., 139 (1994), 395-412.

[M-N] Møller, J. M. and Notbohm, D., Connected Finite Loop Spaces with Maximal Tori, Trans. of A. M. S., 350 (1998), 3483-3504.

[N] Notbohm, D., On the "classifying space" functor for compact Lie groups, J. Lond. Math. Soc., II. Ser. 52, No. 1, 185-198.

[T1] Tits, J., Sur les constantes de structure et le théorème d'existence d'algèbre de Lie semisimple, I.H.E.S. Publ. Math., 31 (1966), 21-55.

[T2] Normalisateurs de Tores: I. Groupes de Coxeter Étendus, J. Algebra, 4 (1966), 96-116.

[W] Whitehead, G. W., Elements of Homotopy Theory, Springer Verlag New York, Grad. Texts in Math., 61 (1978). 
